\title{
PILOT SURVEY OF THE MEDICAL PROFESSIONALS IN BULGARIA ON INTEGRATING EBM TRAINING IN MEDICAL EDUCATION CURRICULUM
}

\author{
Nikoleta Leventi ${ }^{1}$, Antoniya Yanakieva ${ }^{2}$
}

\begin{abstract}
:
INTRODUCTION: Many medical schools all over the world have incorporated evidence-based medicine (EBM) training into their curriculum. According to their results, teaching EBM helps health professionals both to update their knowledge and also to provide better care to their patients in their daily practice while improving the quality of the healthcare system.

OBJECTIVE: The main purpose of this paper is to present the necessity of the integration of EBM training into the medical curriculum in order to improve the quality of education. The introduction of EBM in medical professionals' education in Bulgaria is explored with a pilot study together with the respective opportunities and challenges.

METHODS: About one hundred practicing medical professionals (physicians, dentists, nurses, midwives, and physiotherapists) were included in this pilot study. A paper questionnaire, including three parts (demographic characteristics, attitude towards EBM, application of EBM in everyday clinical practice), was used for collecting data.

RESULTS: Completed questionnaires were received from 84 medical professionals. From the respondents 55 were familiar with the term EBM. The majority (59) of the participants agreed that EBM training would help them in their daily practice. According to 59 of the respondents EBM improves the quality of the provided health and care services to the patients. From the data collected, 65 participants support the necessity of incorporating EBM in their medical education, 9 do not support, and 10 cannot evaluate. Most of the respondents (69) speak and understand a foreign language good enough to read and understand scientific literature without difficulty, as well as the majority (81) of the participants reported computer skills at a good level.
\end{abstract}

CONCLUSIONS: Data from this pilot study demonstrated a positive attitude from medical professionals toward EBM. The majority confirmed the need to integrate EBM training as a discipline into the medical education curriculum.

UDC Classification: 614.2; DOI: http://dx.doi.org/10.12955/cbup.v6.1272

Keywords: EBM training, medical professionals' education.

\section{Introduction}

A review of the available literature from (1996-2006) related to the behavior of doctors in information seeking confirms that traditional methods of face-to-face communication and the use of hard-copy evidence still prevail amongst qualified medical staff in the clinical setting (Davies, 2007).

Presently, the use of the internet as a resource for information of the best quality has increased. Even with the internet as a resource, health professionals still report barriers in searching for the best available evidence. Lack of time, lack of searching skills and sometimes lack of facilities and motivation don't let them to obtain the available information. Here is where evidence-based medicine (EBM) can potentially be utilized to solve the problem by providing medical specialists with better tools in order to achieve their goals (Fourie, 2009).

As a term EBM first appeared in a publication in the American College of Physicians' Journal Club in 1991 by Gordon Guyatt (Guyatt, 1991). According to David Sackett, the most widely quoted definition for EBM states that, "EBM is the conscientious, explicit, and judicious use of current best evidence in making decisions about the care of individual patients" (Sackett et al. 1996). The practice of evidence-based medicine means integrating individual clinical expertise with the best available external clinical evidence from systematic research.

Since then, many medical schools in the world have incorporate EBM into their medical curriculum. The spread of EBM is due to several reasons. Most of them have arisen from medical professionals' daily needs. Clinicians are interested in valid, up to date information in order to make accurate diagnosis and provide optimal treatments through valid prognosis and prevention.

EBM involves three fundamental principles. First, optimal clinical decision-making requires awareness of the best available evidence, which ideally comes from systematic summaries. Second, EBM provides guidance to decide whether evidence is more or less trustworthy and third, evidence alone is never sufficient to make a clinical decision. (Guyatt et al. 2015)

\footnotetext{
${ }^{1}$ Faculty of Public Health / Head of Dept. HTA, Medical University of Sofia, Bulgaria n.leventi@ foz.musofia.bg

${ }^{2}$ Faculty of Public Health / Head of Dept. HTA, Medical University of Sofia, Bulgaria, antoniya.yanakieva@gmail.com
} 
The complete practice of EBM includes the 5A steps:

ASK, ACQUIRE, APPRAISE, APPLY, ASSESS

- Step 1: ASK, to formulate an answerable question (about therapy, diagnosis, prognosis and prevention)

- Step 2: ACQUIRE, search and find the best evidence, which ideally comes from systematic summaries.

- Step3: APPRAISE, critically appraising the evidence for its validity (closeness to the truth), impact (size of effect), and applicability

- Step 4: APPLY, apply the results and integrate them with the clinical expertise and patient's values and preferences.

- Step 5: ASSESS, evaluate the efficacy and effectiveness in $1-4$ steps and find ways to improve them for next time. (Straus et al. 2011)

If you follow an evidence-based approach to clinical decision-making, therefore, all sorts of issues relating to your patients will prompt you to ask questions about scientific evidence, seek answers to those questions in a systematic way and alter your practice accordingly. Hence, EBM requires you not only to read papers but to read the right papers at the right time, and then to alter your behaviour (and, what is often more difficult, influence the behaviour of other people) in the light of what you have found (Greenhalgh T. (2014)).

The major goal of this study is to present the results of a pilot survey on integrating EBM training into the medical education curriculum in Bulgaria in order to help future medical professionals, and to improve the quality of education.

\section{Methods}

The study is ongoing. In this article, we present preliminary results from a pilot phase of the study that we conducted from October to December of 2017. These results provide information about the necessity of including the EBM training discipline into the medical curriculum and also, a positive attitude towards the EBM approach. For the study, we used a questionnaire among physicians, dentists, nurses, midwives and physiotherapists. The design of the questionnaire consisted of three parts and the total number of questions was 47 . The participation was anonymous, and all the respondents were volunteers.

The first part of the questionnaire included demographics features and asked respondents to describe personal and professional characteristics such as: age, nationality, medical specialty and sub specialty, where they work, year of graduation, years of practice, computer skills, etc. The total number of questions in this part was 14. The second part of the questionnaire included questions about the attitude of the medical professionals towards EBM. This part of the questionnaire included 21 questions. The third (last) part of the questionnaire asked participants about the application of EBM in their daily clinical practice. The number of questions in the third part was 12 .

Most of the questions were formulated as closed questions. Statements related to closed questions were given a range for the answer of:

- Yes / No

- Yes, No, "I cannot evaluate"

- "strongly agree", "agree", "I cannot evaluate", "disagree", and "strongly disagree".

Apart from the closed questions, the first part contained 2 open questions, the second 1 , and in the third part there were 2 open questions.

This paper partially presents the results of the first and second parts of the questionnaire.

\section{Results}

Completed questionnaires were received from 84 medical professionals. The survey is ongoing, thus further results will be presented when the full survey is concluded, approximately the second half of 2018.

\section{DEMOGRAPHICS RELATED RESULTS}

Data collected from 84 participants demonstrates that the majority of them were female 46 , and according to their answers regarding their age, 32 were between (35-40) years, 30 were older than 40 
years, 11 were between (30-35) years, 8 were between (25-30) years, and 3 of them were about 25 . Related to their specialty, dentists accounted for 30 of the respondents, physicians 29, nurses 13 , midwives 11 and physiotherapist only 1 . Information about their work experience shows that the majority (34) of medical professionals have more than 40 years of experience, 17 of them had experience between 11-20 years, 18 of the responders have experience between 6 and 10 years and 15 of the respondents had experience of five or less years. The distribution of the responses to the question related to the university they graduated from. 28 of the respondents graduated from the Medical University of Sofia, 14 from the medical university of Varna, 13 from the medical university of Plovdiv, and 13 from other institute(s).

It is worth mentioning the last two questions of the demographics characteristics consider the knowledge of foreign language and computer skills of the participant. The majority of the respondents (69) speak and understand a foreign language good enough to read and understand scientific literature without difficulty as well as the majority (81) of the participants reported computer skills at a good level (Table 1). In general, these results demonstrate that such skills and knowledge are useful in order for medical professionals to be effective in researching EBM processes (Fig. 1, Fig. 2).

\begin{tabular}{|l|c|c|}
\hline \multicolumn{2}{|l|}{ Table 1: Demographics related results } \\
\hline \multicolumn{1}{|c|}{ Characteristics } & $\begin{array}{c}\text { Total number } \\
\text { (YES) }\end{array}$ & $\begin{array}{c}\text { Total number } \\
\text { (No) }\end{array}$ \\
\hline Total number participated 84 & & \\
\hline $\begin{array}{l}\text { Do you speak and understand a foreign language good enough to use } \\
\text { scientific literature without difficulty? }\end{array}$ & 69 & 15 \\
\hline Do you have computer skills? & 81 & 3 \\
\hline
\end{tabular}

Source: Author

\begin{tabular}{|l|}
\hline Figure 1: Participants who speak and understand a foreign language good enough to use scientific \\
literature without difficulty. \\
\hline \\
\hline Source: Author
\end{tabular}

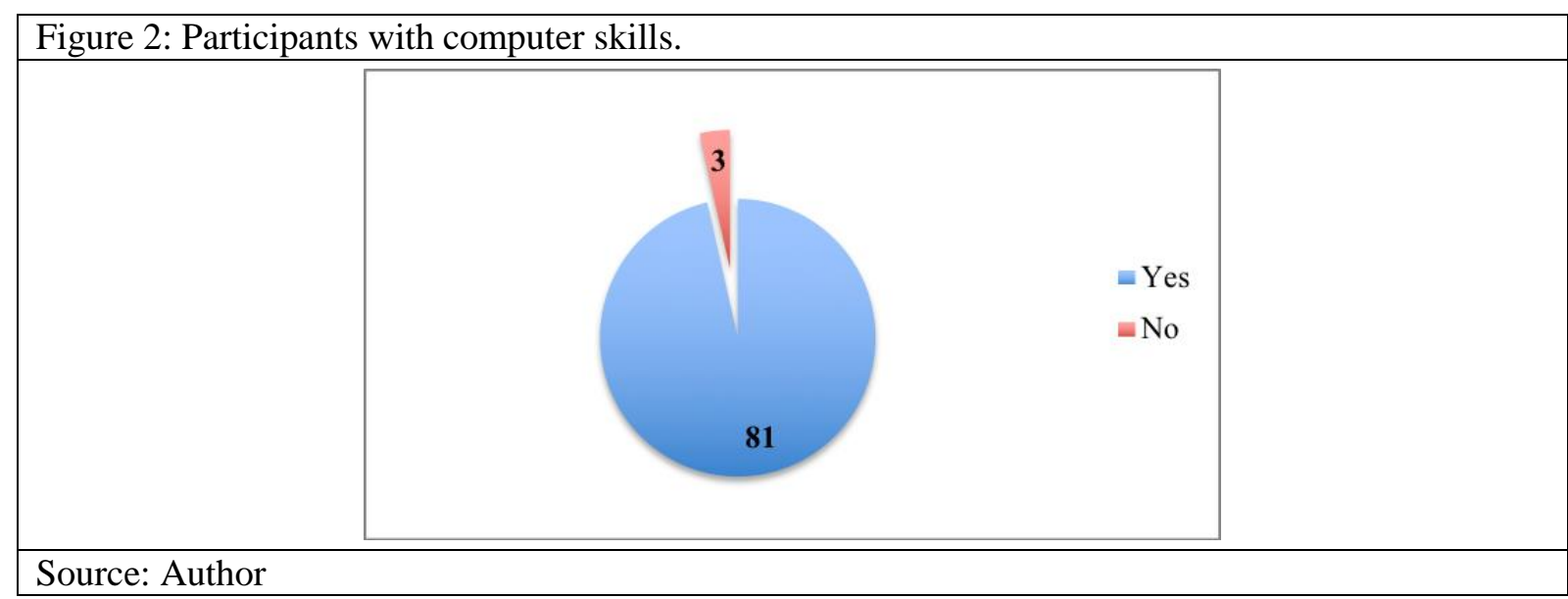




\section{ATTITUTE TOWARDS EBM RELATED RESULTS}

The second part of the questionnaire included 21 questions, and all were related to the attitude towards EBM. The feedback of the questionnaire demonstrates that 55 of the respondents were familiar with the term EBM; 28 of them were familiar with the term through their university education, 20 through colleagues, 19 from medical journals, 12 from conferences, 7 from seminars and 2 from other resources.

The interesting point of the second part is that despite the fact that half of the respondents (42) are familiar with the medical information resources like (Cochrane database of systematic reviews, Medline etc.) only 20 used them in their practice once a week, 12 once a month, 10 several times a year, and 41 of the participants do not use them at all.

Of the respondents 40 stated they strongly agree that EBM leads to a rapid updating of knowledge, 22 stated they agree, 19 they cannot evaluate, 2 do not agree, and only 1 strongly disagrees (Fig. 3).

The majority (59) of the participants agreed that EBM training would help them in their daily practice (Fig. 4). According to 59 of the respondents EBM improves the quality of the provided health and care services to the patients (Fig. 5). Also 52 of the medical professionals believe that the reduction in health care costs is directly related to application and understanding of EBM. The distribution of responses shows that 32 of the responders are not familiar with the term health technology assessment (HTA) and also only 34 support that EBM improves the HTA process, 2 that it does not improve it and 48 they cannot evaluate.

Even though most of the medical providers are familiar with the term EBM only 32 know the steps of the EBM process, 38 are familiar with the hierarchy of evidence in EBM and only 30 are familiar with the strength of recommendations.

It is worth mentioning that the majority of the respondents (59) report that they could use it in their future work but under some circumstances. In order to do so, it is necessary for 42 of them to undergo additional training, 28 require access to medical information resources, and for 18 is necessary to have access to the internet in their workplace.

Despite that, the majority of the participants (36) are familiar with the term randomized clinical trials (RCT), 19 are strongly familiar, 12 strongly unfamiliar, 11 unfamiliar, and 6 cannot evaluate, only 2 of the respondents could give a correct definition of RCT. Very interesting are the results related to their critical appraise on the quality of RCT. Many of the respondents (38) believe that they can assess the quality of RCT, while 24 that they cannot assess and 22 cannot evaluate.

From the data collected, 65 participants support the necessity of incorporating EBM in their medical education, 9 do not support it, and 10 cannot evaluate (Fig. 6). According to the answers many of the respondents (24) believe that they do not effectively use the opportunities of EBM in Bulgaria.

\begin{tabular}{|l|l|l|}
\hline Figure 3: Participants' opinion whether EBM leads to a rapid updating of knowledge. \\
\hline
\end{tabular}




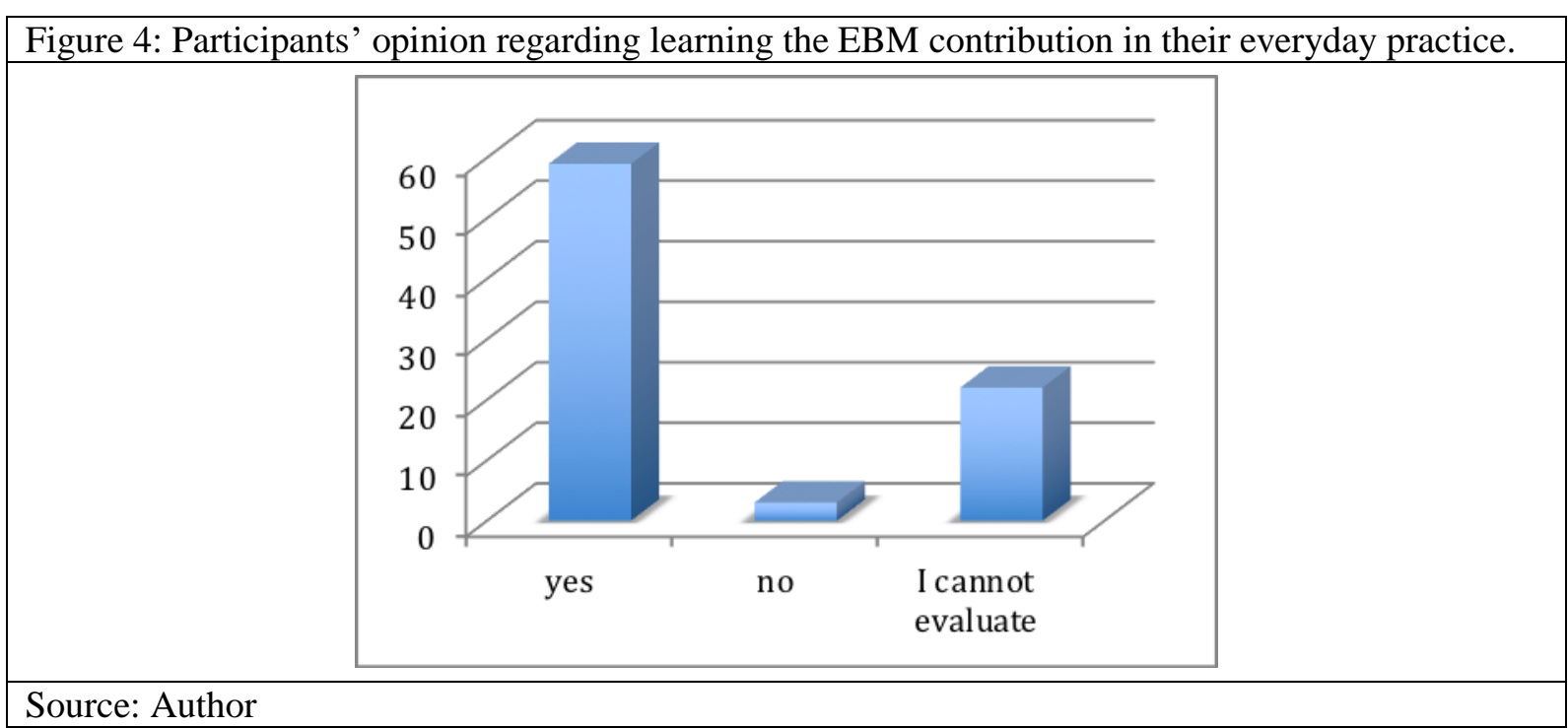

Figure 5: The practice of EBM improves the quality of patient care - participants' answers.

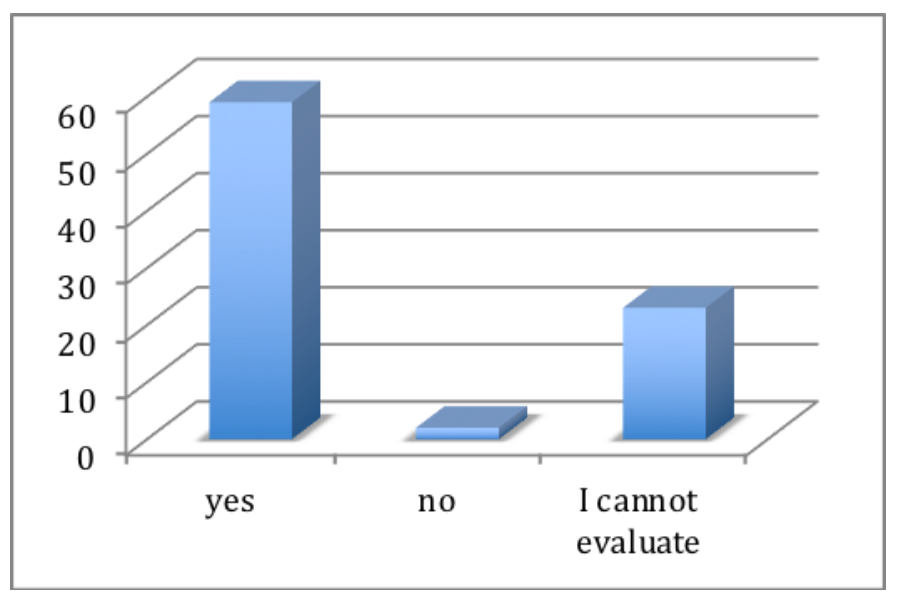

Source: Author

Figure 6: Participants' opinion on the necessity of the integration of an EBM training discipline in their medical curriculum.

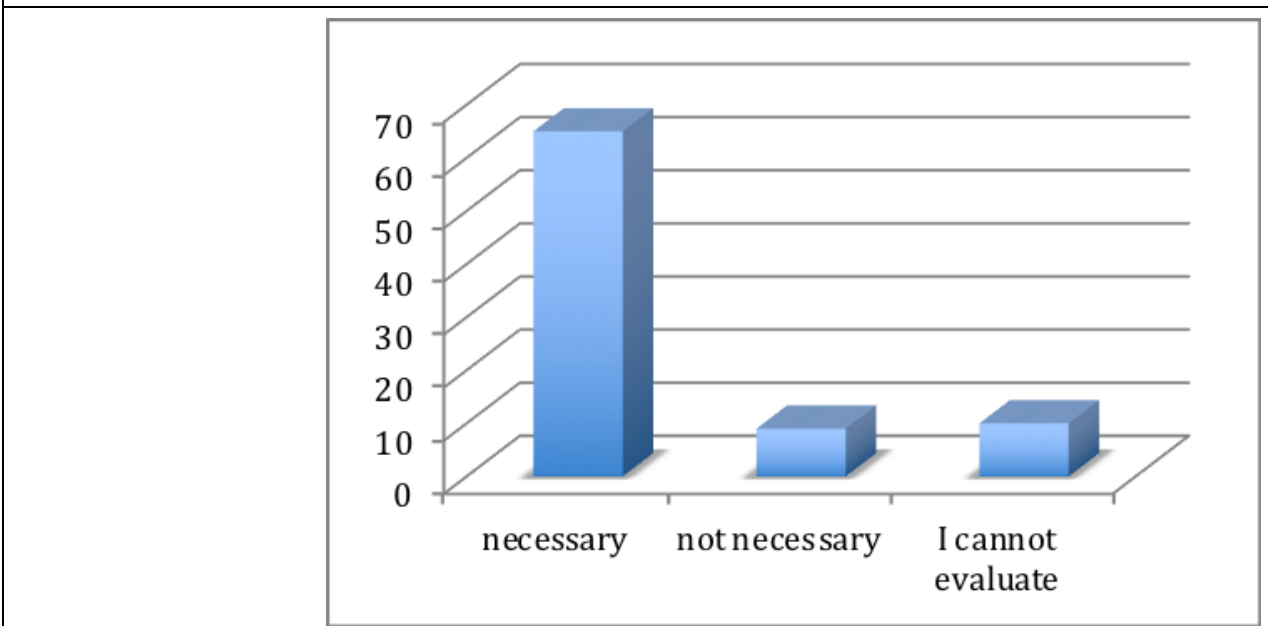

Source: Author 


\section{Conclusion}

The aim of this study is to introduce EBM into the education of medical professionals. Data from this pilot phase of the study demonstrated a positive attitude from medical professionals toward EBM. The majority confirmed the need to integrate EBM training into the medical education curriculum. This integration will help medical professionals to understand the steps of the EBM process, will make them more confident to assess the quality of evidence and will help health care providers to improve the quality of patient care in their daily practice. In addition, incorporation of EBM training will inform medical professionals about the importance of evidence in the HTA process, in order to make our health systems more effective.

\section{References}

Davies K. (2007), The information-seeking behavior of doctors: a review of the evidence, Health Information and Libraries Journal 2007; 24(2): 78-94.

Fourier I. (2009), Learning from research on the information behaviour of health care professionals: a review of the literature 2004-2008 with a focus on a motion, Health Information and Libraries Journal 2009; 26(3): 171-86.

Guyatt G. (1991), Evidence-based medicine. ACP J Club (Ann Intern Med), 1991; 114(suppl. 2):A-16.

Sackett DL, Rosenberg WM, Gray J. (1996), Evidence based medicine: what it is and what it isn't. BMJ: British Medical Journal 1996; 312 (7023): 71.

Guyatt G., Rennie D., O. Meade M., Cook D. (2015), Users' Guide to medical Literature, 3-rd edition, Mc Graw Hill Education, New York, ISBN: 978-0-07-180872-9, 2015.

Straus S., Glasziou P., Richarson W.S., Haynes R. B. (2011), Evidence-based medicine: How to practice and teach it, 4-th edition, Churchill Livingstone Elsevier, Edinburg, ISBN: 978-0-7020-3127-4, 2011.

Greenhalgh T. (2014), How to read a paper: The basics of Evidence-Based Medicine, 5-th edition, Willey Blackwell, BMJ Books, UK, ISBN: 978-1-118-80096-6 (pbk.), 2014. 